\title{
A Modified Approach Based On SLM for OFDM PAPR Reduction Using Time Domain Sub-Block Conversion Matrix
}

\author{
Yasmeen Z.Attar \\ PG Student, EXTC, Saraswati \\ College of Engineering, \\ Kharghar, Navi Mumbai
}

\author{
Sheetal Bhujade \\ Assistant Professor \\ Saraswati College of \\ Engineering, Kharghar, Navi \\ Mumbai
}

\begin{abstract}
OFDM is a digital transmission method developed to meet the increasing demand for higher data rates in communications which can be used in both wired and wireless environments. This thesis describes the issue of the PAPR in OFDM which is a major drawback, and presents new and variations to existing algorithms to reduce it. The effect on system performance in terms of the BER and Power Spectral Density is simulated for an OFDM transceiver with a saturated High Power Amplifier. A modified SLM approach based on time-domain sub-block conversion matrices to reduce the computational complexity due to multiple IFFTs. This scheme is modified from the conversion matrix scheme. By dividing the frequency-domain signals into multiple sub-blocks, the number of the valid conversion matrices can be increased, and thus more candidate signals are available for PAPR reduction. By applying proposed scheme, the number of candidate signals can be increased from 12 in the original conversion matrix scheme to 28 and 128 for the two sub block and four-sub block cases, respectively. The scheme will provide improved PAPR reduction performance.
\end{abstract}

\section{Keywords}

Orthogonal Frequency Division Multiplexing (OFDM), peakto-average power ratio (PAPR), selective mapping (SLM), Inverse Fast Fourier Transformation (IFFT), Modified SLM (MSLM), Time Domain Sub-Block Conversion Matrix (TSCM-SLM).

\section{INTRODUCTION}

Orthogonal frequency division multiplexing (OFDM) is a promising technique for high-speed data transmission, because of its high spectral efficiency and robustness against multipath fading. It has been adopted in many standards, such as digital radio audio/video broadcasting (DAB/DVB) systems, IEEE $802.11 \mathrm{a} / \mathrm{g}$, IEEE 802.16 and 3GPP long-term evolution (LTE) systems. In OFDM systems, peak-to-average power ratio (PAPR) reduction is a very important issue since high PAPR results in signal distortion while passing through a high power amplifier or reduces the power-amplification efficiency. In order to improve the efficiency of a power amplifier, numerous techniques have been proposed to reduce the PAPR of OFDM time-domain signals [1]-[3]. The
Selective mapping (SLM) scheme is the most popular and effective PAPR-reduction approach [3].

However, its complexity is usually too high to be acceptable in practical applications. In order to reduce the complexity of the conventional SLM scheme, Wang et al. proposed a modified SLM method by using low-complexity conversion matrices (hereinafter referred to as the MSLM scheme), which can relieve the requirement of multiple inverse fast Fourier transformation (IFFT) operations [4]-[6].To reduce the complexity, the real part and imaginary part of each element in the conversion matrices must be restricted to the three values $\{0,+/-1\}$ therefore; the generation of a candidate signal involves no complex additions and multiplications. Moreover, the phase factor on each subcarrier is restricted to the four values $\{+/-1,+/-j\}$. Unfortunately, by applying MSLM, the number of valid candidate signals is restricted to 12 , leading to a strictly limited PAPR reduction performance. In order to reduce the complexity of the conventional SLM scheme, Wang. Proposed a modified SLM method by using lowcomplexity conversion matrices which can relieve the requirement of multiple inverse fast Fourier transformation (IFFT) operations [4]-[8].If more candidate signals are desired, some other deficient conversion matrices can be generated at the cost of degradation in the BER performance [8]-[13].

\section{SYSTEM MODEL}

The considered OFDM system is assumed to comprise $\mathrm{N}$ subcarriers. Let $\mathrm{X}=\left[\begin{array}{llll}X 0 & X 1 & X 2 & X N-1\end{array}\right] T$ denote the frequency domain data vector transmitted in an OFDM symbol. The corresponding time-domain baseband signal is represented as

$$
\mathrm{x}(\mathrm{t})=\frac{1}{\sqrt{N}} \sum_{k=0}^{N-1} X k \exp (j 2 \pi k \Delta f t), 0 \leq t \leq T
$$

Where $\Delta \mathrm{f}$ is the subcarrier spacing and $\mathrm{T}$ is the OFDM symbol duration. The PAPR of $x(t)$ is defined as the ratio of the peak power to the average power of $x(t)$ and can be written

$$
\begin{aligned}
& \text { PAPR }=\frac{\text { Peak power }}{\text { average power }} \\
& P A P R=\max _{0 \leq t \leq T}|x(t)|^{2} / E\left[|x(t)|^{2}\right]
\end{aligned}
$$


For discrete-time analysis, the discrete-time version of $\mathrm{x}(\mathrm{t})$ as $\mathrm{xk}=\mathrm{xk}(\mathrm{T} / \mathrm{LN}), 0 \leq \mathrm{k} \leq \mathrm{LN}$, where $\mathrm{L}$ is the over-sampling factor and typically assumed to be 4 [1]. Accordingly, the PAPR can be rewritten as

$$
P A P R=\max _{0 \leq k \leq L N}\left|x_{\iota}\right|^{2} / E\left[\left|x_{\downarrow}\right|^{2}\right.
$$

\section{PAPR REDUCTION METHODS FOR OFDM SIGNAL SELECTED MAPPING (SLM)}

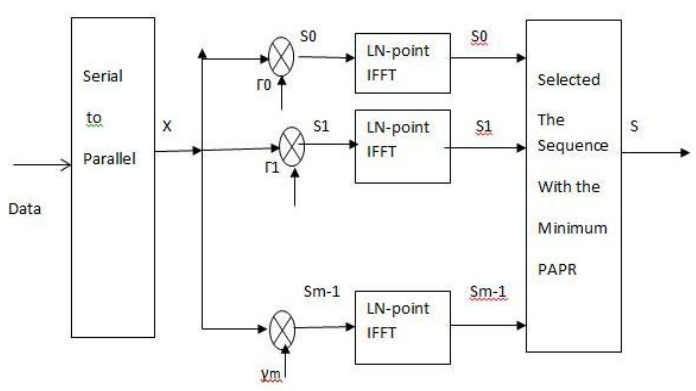

Fig.1: Conventional SLM

In SLM, an input symbol sequence is multiplied by each of the predetermined sequences, called phase sequences, to yield alternative input symbol sequences. Each of these alternative input symbol sequences is inverse fast Fourier transformed (IFF), and then, the one with the lowest PAPR is selected for transmission. In the conventional SLM scheme, $M$ statistically independent phase sequences are generated, and then multiplied by the frequency-domain data sequence $\mathrm{X}$ to produce the $M$ independent candidate signals. Through IFFT transformations, the candidate signal with the lowest PAPR is selected for transmission, as shown in Fig. 1. Let the frequency-domain candidate signal $\mathrm{S} 0=\mathrm{X}$ be the original data vector and the corresponding time-domain signal be $\mathrm{s} 0=\mathrm{F}-1 \mathrm{~S} 0$ $=\mathrm{F}-1 \mathrm{X}$, where $\mathrm{F}-1$ represents the matrix of IFFT. We denote the random generated phase vector as

$\gamma m=\left[\begin{array}{lllll}b 0 X 0 & b 1 \times 1 & \ldots \ldots & \ldots & b n X n\end{array}\right] T$

For $\mathrm{m}=0, \ldots, \mathrm{M}-1$, and the frequency-domain candidate signals as

$$
\begin{aligned}
S m & =R m X_{=}\left[\begin{array}{lll}
b 0 X 0 & b 1 X 1 & b n X n
\end{array}\right]_{T} \\
R m & =\left[\begin{array}{ccc}
b 0 & 0 & 0 \\
0 & b 1 & 0 \\
0 & 0 & b n
\end{array}\right]
\end{aligned}
$$

\subsection{Modified SLM}

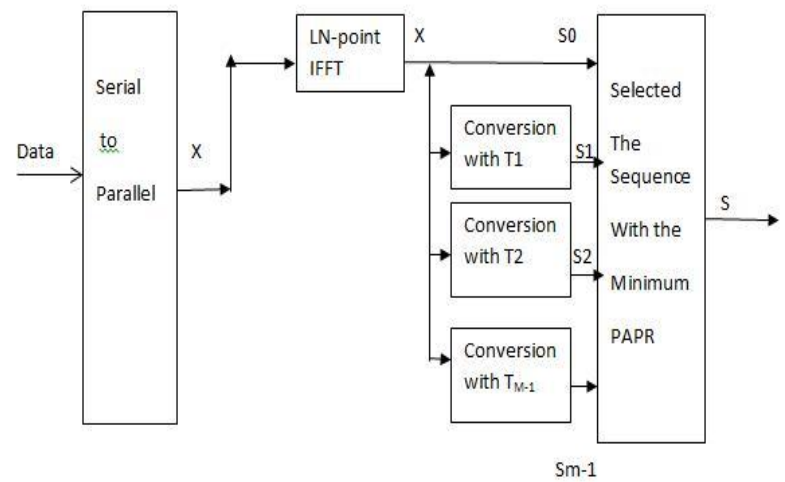

Fig 2: Modified SLM

To reduce the complexity, the real part and imaginary part of each element in the conversion matrices must be restricted to the three values $\{0,+/-1\}$. Therefore, the generation of a candidate signal involves no complex multiplications. The architecture of the scheme is shown in Fig. 2. The timedomain signal of the original data sequence is first generated by applying IFFT on $\mathrm{X}$, i.e., $\mathrm{x}=\mathrm{s} 0=\mathrm{F}-1 \mathrm{~S} 0$. The conversion matrices are denoted as $\mathrm{Tm}$, for $\mathrm{m}=0 \ldots \mathrm{M}-1$, and the candidate signals are obtained by This restriction can benefit the phase compensation process at the receiver, since no complex multiplication is required.

\subsection{TSCM-SLM:}

To improve the efficiency with the help of the TSCM-SLM scheme reduce the value of PAPR where in order to obtain a sufficient number of candidate signals while maintaining a very low computational complexity without causing any degradation in the BER performance. The basic concept of the TSCM-SLM scheme is to divide the frequency domain data sequence into multiple sub-blocks by using interleaved partition. In other words, each sub-block contains the data symbols upon subcarriers with equally-spaced indices.

\subsubsection{Two Sub-Block TSCM-SLM:}

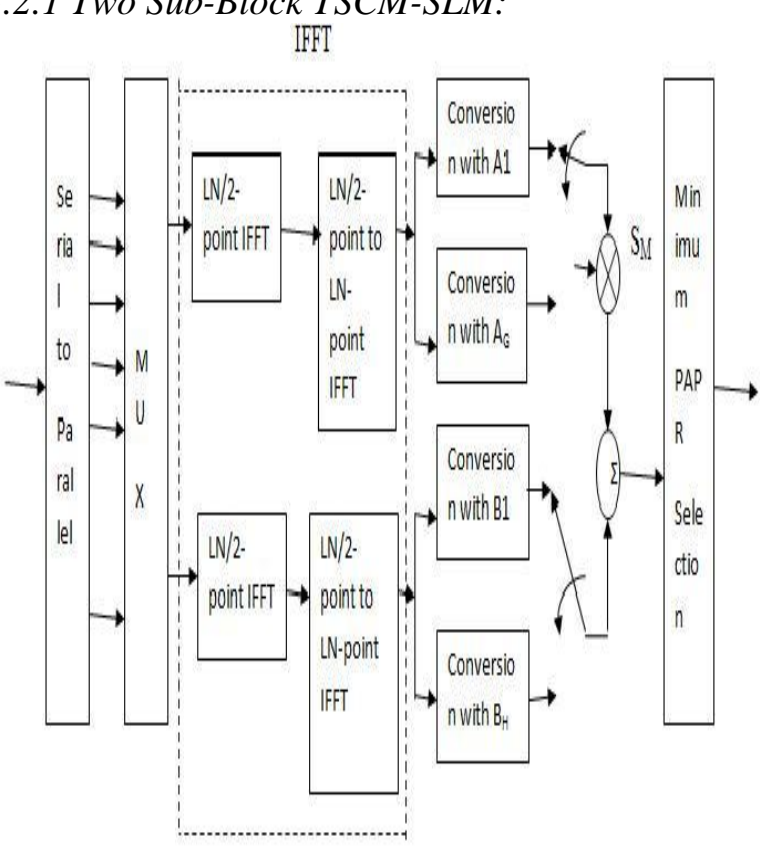

Fig.3: Two Blocks TSCM-SLM

Shown in Fig. 3 is the architecture of the proposed two sub block TSCM-SLM scheme. The input data sequence is divided into two sub-blocks with length $\mathrm{N} / 2$. In other words, the N/2 even-indexed subcarriers are grouped in one sub block $\mathrm{Xe}$ and the N/2 odd-indexed subcarriers are grouped in the other one Xo. After over-sampling and passing through the

LN/2 -point IFFT operation, we obtain the time-domain signals with the length equal to LN/2. Note that the length of the partial candidate signals is equal to $\mathrm{LN} / 2$ and can be extended to length LN easily by applying the basic concepts of FFT [7]. The resultant time-domain signals are represented as $\mathrm{Xe}$ and xo, respectively. Then, $\mathrm{Xe}$ and xo are respectively multiplied by the conversion matrices $\mathrm{Ag}$ and $\mathrm{Bh}$ to obtain the 
partial candidate signals. Finally, a candidate signal sm is obtained by summing up the two extended partial candidate signals se, $m$ and so,m.

To guarantee that the complexity of the proposed TSCM-SLM scheme is not higher than that of the MSLM scheme, so restrict the total number of involved complex vector additions, including the operation of sub-block conversions and the summation of partial candidate signals, to be less than three.

\subsubsection{Four Sub-Block TSCM-SLM:}
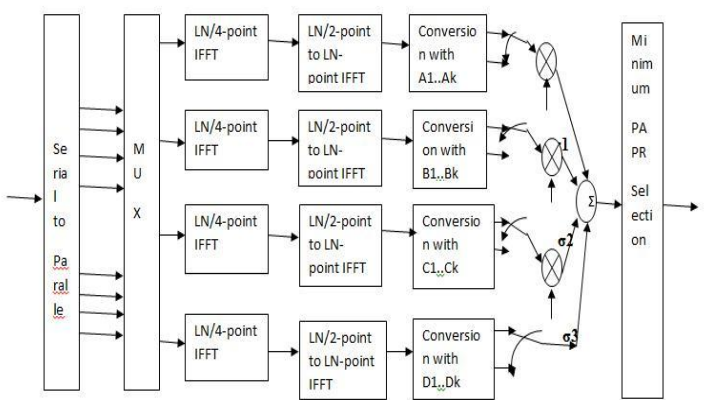

Fig.4: Four Blocks TSCM-SLM

In order to obtain more available candidates, divide the frequency-domain OFDM signal into more sub-blocks like four sub-blocks Figure 4 shows the architecture of the foursubblock TSCM-SLM scheme. In this approach, the modified constraint to restrain the computational complexity is reduced by three numbers of complex additions.

\section{ANALYSIS OF COMPUTATIONAL COMPLEXITY}

The first column vectors of these sub-block conversion matrices, as well as the corresponding partial phase sequences, are obtained by computer search for two subblocks and shown in TABLE I. Note that combining the partial phase vectors of all sub-blocks yields the overall phase rotation vector.

Table 1.

\begin{tabular}{|c|c|c|}
\hline & First Column Vectors & Phase Vectors \\
\hline \multirow{4}{*}{ A1 } & $\theta 1=\left[\begin{array}{lllllll}1 & 0 & 0 & 0 & 0 & 0\end{array}\right] \mathrm{T}$ & 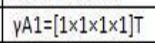 \\
\hline & $\theta_{22}=\left[\begin{array}{llllll}0 & 10 & 0 & 0 & 0 & 0\end{array}\right] \mathrm{T}$ & vA2 $=[1 x-j x-1 x j] T$ \\
\hline & 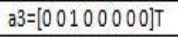 & $\gamma A 3=[1 x-1 \times 1 x-1] T$ \\
\hline & $84=\left[\begin{array}{llllllll}0 & 0 & 1 & 0 & 0 & 0\end{array}\right] \mathrm{T}$ & $\gamma A 4=[1 \times 1 \times-1 x-1] T$ \\
\hline \multirow{6}{*}{ B1 } & $\mathrm{b1}=\left[\begin{array}{llllll}1 & 0 & 0 & 0 & 0 & 0\end{array}\right] \mathrm{T}$ & $\gamma B 1=[1 \times 1 \times 1 \times 1] T$ \\
\hline & 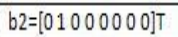 & $\gamma B 2=[1 x-j x-1 x j] T$ \\
\hline & 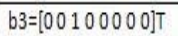 & $\gamma B 3=[1 x-1 \times 1 x-1] T$ \\
\hline & $\mathrm{b} 4=\left[\begin{array}{llllll}0 & 0 & 1 & 0 & 0 & 0\end{array}\right] \mathrm{T}$ & $\gamma B 4=[1 \times 1 x-1 x-1] T$ \\
\hline & 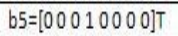 & $\gamma B 5=[1 \mathrm{x}-\mathrm{j} \mathrm{x}-1 \mathrm{1}-1] \mathrm{T}$ \\
\hline & $\mathrm{b} 6=[00010000] \mathrm{T}$ & $\gamma B 6=[1 \times 1 \times-1 \times-j] T$ \\
\hline
\end{tabular}

Table 2.

\begin{tabular}{|l|c|c|}
\hline & $\begin{array}{l}\text { Average number of multiplica } \\
\text { tions per candidate signal }\end{array}$ & $\begin{array}{l}\text { Average number of additions } \\
\text { per candidate signal }\end{array}$ \\
\hline Conventional SLM & $\left(\frac{L N}{2}\right) \log 2(L N)$ & $(L N) \log 2(L N)$ \\
\hline MSLM for M $=12$ & $\left(\frac{L N}{24}\right) \log 2(L N)$ & $\left(\frac{L N}{12}\right) \log 2(L N)+2.75 L N$ \\
\hline $\begin{array}{l}\text { TSCM-SLMM }=28 \text { (two sub- } \\
\text { blocks) }\end{array}$ & $\left(\frac{L N}{56}\right) \log 2(L N)$ & $\left(\frac{L N}{28}\right) \log 2(L N)+1.57 L N$ \\
\hline $\begin{array}{l}\text { TSCM-SLMM }=128 \text { (four sub- } \\
\text { blocks) }\end{array}$ & $\left(\frac{L N}{256}\right) \log 2(L N)$ & $\left(\frac{L N}{128}\right) \log 2(L N)+2.98 L N$ \\
\hline
\end{tabular}

The computational complexity of different schemes is shown in TABLE 2 with the oversampling factor L. In the SLM scheme, the generation of a candidate signal re- quires an LNpoint IFFT operation. In MSLM, only one LN- point IFFT operation is required for the generation of the $M=12$ candidate signals. Similarly, in our proposed scheme, the complexity of the blocks surrounded by dashed lines, as shown in Fig. 3 and Fig. 4, is the same as an LN-point IFFT operation, except for the final summation operation. As a result, we have the average numbers of complex multiplications and additions as shown in TABLE 2.

\section{SIMULATION RESULTS}

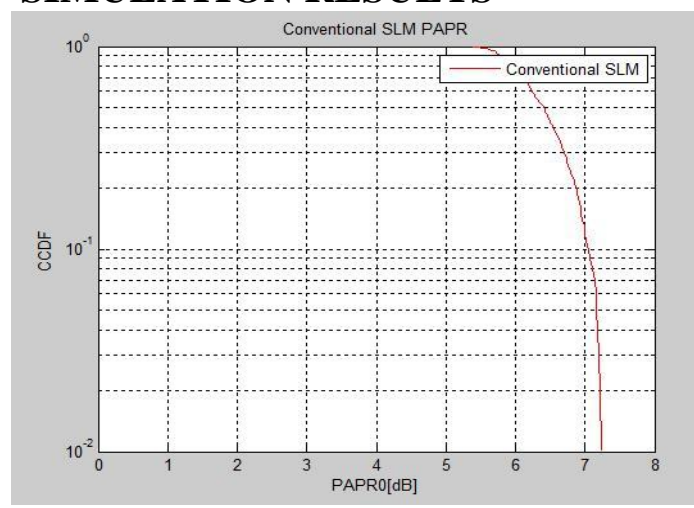

Fig.5: Result of Conventional SLM

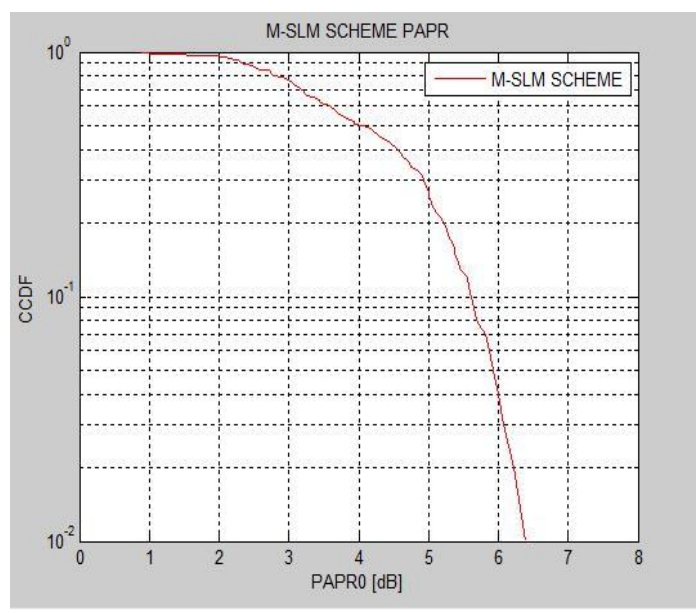

Fig.6 Result of MSLM 


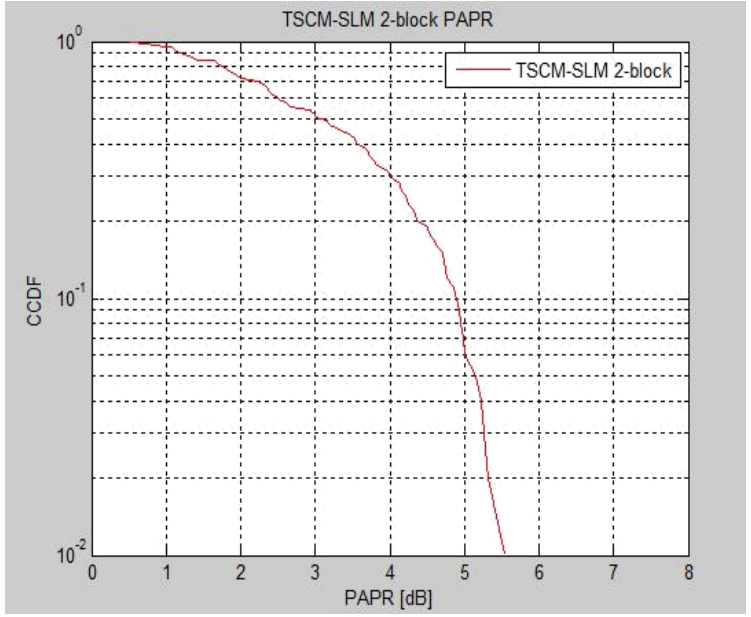

Fig.7: Result of two sub blocks TSCM-SLM

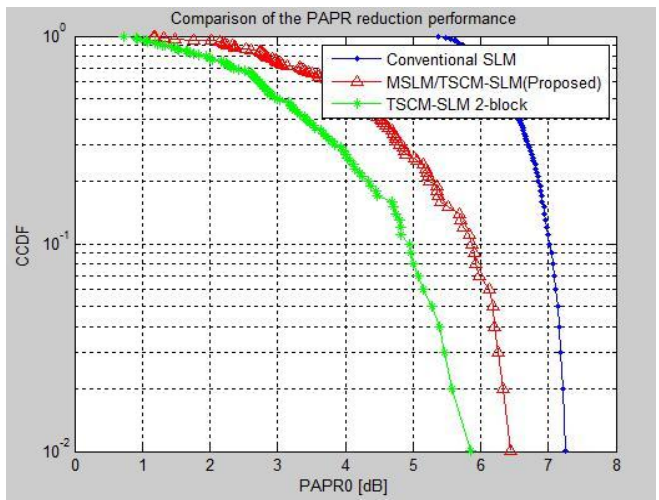

Fig.8: Comparison between Conventional, MSLM and two subblocks

The complementary cumulative distribution function (ccdf), defined as the probability that the PAPR value is smaller than a specific value, is used to evaluate the PAPR reduction performance. In the simulations, the number of subcarriers $\mathrm{N}$ is set to be 256,512 or 1024 , and the 16-QAM modulation scheme is adopted. Fig.5 shows the conventional SLM, Fig.6 MSLM and Fig.7 shows the result for conventional, modified and two subblocks TSCM-SLM respectively.Fig.8 shows the performance comparison of the proposed TSCM-SLM scheme and the MSLM scheme. Since the number of available candidate signals by applying TSCM-SLM is significantly larger than that obtained by MSLM,the performance of TSCM-SLM is substantially better than that of MSLM.

\section{CONCLUSION}

In this paper, we have proposed low-complexity conversions for LN -point IFFT computation in the SLM approach with M candidate signals, where $\mathrm{L}$ is the oversampling factor. These conversions use one IFFT output signal to produce other IFFT output signals, i.e., the candidate signals. We have utilized them to replace the IFFT blocks in the conventional SLM approach and proposed two new SLM schemes. Both of the proposed SLM schemes have much lower complexity than the conventional one. Computer simulation results have shown that, as compared to the conventional SLM scheme. By using more different randomly generated vectors along with more IFFT blocks in TSCM-SLM scheme, we can generate more candidate signals for transmission and thus achieve better PAPR reduction performance. With the low-complexity and good-performance features, the proposed SLM schemes are rather attractive for using OFDM-based communication systems. Compared to MSLM, applying the proposed scheme can result in not only more valid candidate signals, but also less computational complexity per candidate signal. The gains in PAPR reduction performance are about $0.2 \mathrm{~dB}$ and $0.9 \mathrm{~dB}$ for the two-subblock.

Since the number of candidate is increased by applying, more side information is also needed as compared to MSLM. Again for the same size of side information, the proposed TSCMSLM scheme can significantly reduce computational Complexity as compared to the conventional SLM. It also improve the system performance by using Four subblocks TSCM-SLM scheme in future.

\section{ACKNOWLEDGMENTS}

I would like to thanks my guide Prof.Sheetal Bhujade for her valuable guidance and suggestions while writing this paper.

\section{REFERENCES}

[1] A. V. Oppenheim, R. W. Shafer and J. R. Buck, Discrete-Time Signal Processing, 2/e.Prentice Hall, 1999.

[2] R. W. Bauml, R. F. H. Fischer and J. B. Huber, "Reducing the peak-to average power ratio of multicarrier modulation by selective mapping," Electron. Lett., vol. 32, no. 22, pp. 2056- 2057, Oct. 1996.

[3] A. D. S. Jayalath and C. Tellambura, "Reducing the peak-to- average power ratio of orthogonal frequency division Multiplexing signal through bit or symbol interleaving," IETEletronics Letters, vol. 36, no. 13, pp.1161-1163, Jun. 2000.

[4] C.-L. Wang,M.-Y.Hsu and Y.Ouyang, "A low Complexity peak-to average power ratio reduction technique For OFDM systems," in Proc. IEEE GLOBECOM, Dec. 2003, vol. 4, pp.2375-2379.

[5] A. D. S. Jayalath and C. R. N. Athaudage, "On the PAPR reduction of OFDM signals using multiple signal representation," IEEE Commun. Letters, vol. 8, no. 7, pp. 425-427, July 2004.

[6] C.-L. Wang and Y. Ouyang, "Low-complexity selected mapping schemes for peak-to-average power ratio reduction in OFDM systems," IEEE Trans. Signal Processing, vol. 53,no. 12, pp. 4652-4660, Dec. 2005.

[7] G. Lu, P. Wu and C. Carlemalm-Logothetis, "Enhanced Interleaved partitioning PTS for peak-to-average Power ratio reduction in OFDM systems," Electron. Lett., vol. 42, no.17,pp. 983-984, Aug. 2006

[8] C.-L. Wang and S.-J. Ku, "Novel conversion matrices forSimplifying the IFFT computation of an SLM -based PAPR reduction scheme for OFDM systems," IEEE Trans Commun., vol.57, no. 7, pp. 1903-1907, July 2009

[9] Dae-Woon Lim, Jong-Seon No,"A New SLM OFDM Scheme With Low complexity for PAPR Reduction", Member, IEEE, Chi-Woo Lim, and Habong Chung, Member, IEEE. .

[10] Shazia Shireen, Jyoti Asnani, Prof. Ravi Shankar Mishra and Prof.Rajesh Nema, "A Comparison of Peak to Average Power Reduction Schemes for OFDM", Electronics \& Comm.Engineering NIIST Bhopal. 
International Journal of Computer Applications (0975 - 8887) International Conference on Advances in Science and Technology (ICAST-2014)

[11] Sudesh Gupta Prof. Ravi Shankar Mishra, Prof. Rajesh Nema, "PAPR Reduction in OFDM via Separation of Complex Baseband Signal", Dept. of Electronics \& Comm.NIIST, Bhopal.

[12] M. Michel TERRE Professor, CNAM, Paris M. Mischa DOHLER Chercheur, CTTC, Barcelone, "Peak to
Average Power Ratio Analysis and Reduction of Cognitive Radio Signals" Université de Rennes.

[13] Ramanjeet Kaur, Charanjit Singh, Amandeep Singh, "Performance Analysis of Different Selected Mapping Schemes for OFDM Systems" Department ofElectronics and Communication Engineering. Punjabi University Patiala, India, IJERA. R. J. Vidmar. (August 1992 